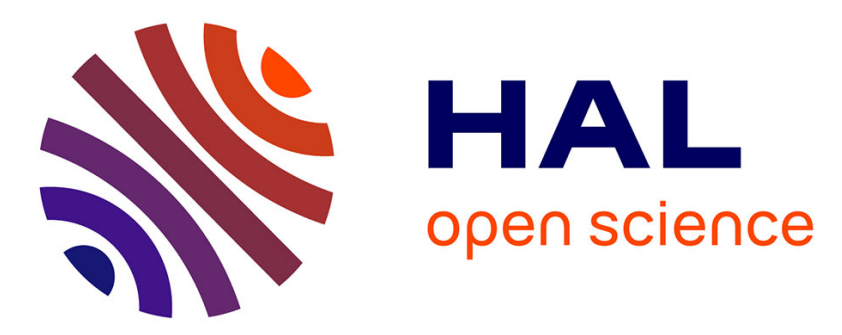

\title{
A Hybrid Adaptive Coding and Decoding Scheme for Multi-hop Wireless Sensor Networks
} Imad Ez-Zazi, Mounir Arioua, Ahmed El Oualkadi, Pascal Lorenz

\section{To cite this version:}

Imad Ez-Zazi, Mounir Arioua, Ahmed El Oualkadi, Pascal Lorenz. A Hybrid Adaptive Coding and Decoding Scheme for Multi-hop Wireless Sensor Networks. Wireless Personal Communications, 2016, pp.1-17. 10.1007/s11277-016-3763-1 . hal-01510173

\section{HAL Id: hal-01510173 \\ https://hal.science/hal-01510173}

Submitted on 19 Apr 2017

HAL is a multi-disciplinary open access archive for the deposit and dissemination of scientific research documents, whether they are published or not. The documents may come from teaching and research institutions in France or abroad, or from public or private research centers.
L'archive ouverte pluridisciplinaire HAL, est destinée au dépôt et à la diffusion de documents scientifiques de niveau recherche, publiés ou non, émanant des établissements d'enseignement et de recherche français ou étrangers, des laboratoires publics ou privés. 


\title{
A hybrid adaptive coding and decoding scheme for multi-hop wireless sensor networks
}

\author{
Imad Ez-zazi · Mounir Arioua · Ahmed \\ El Oualkadi · Pascal Lorenz
}

Received: date / Accepted: date

\begin{abstract}
In this paper, we have proposed a hybrid adaptive coding and decoding scheme for multi-hop wireless sensor networks (WSNs). Energy consumption and transmission reliability are used as performance metrics for multi-hop communications in WSNs. The presented scheme takes into account distance, channel conditions and correction codes performance to decide coding and decoding procedure, and considers Reed Solomon (RS) code and Low Density Parity Check (LDPC) code to provide error protection on the transmitted data. The proposed approach aims to reduce the decoding power consumption and to prolong the lifetime of the network as well as improve the reliability of the transmission. Simulation results show that our performed scheme enhances both energy efficiency and communication reliability of multi-hop sensor networks.
\end{abstract}

Keywords WSN $\cdot$ ECC $\cdot \mathrm{LDPC} \cdot \mathrm{RS} \cdot \mathrm{Multi-hop} \cdot \mathrm{MTE}$

\section{Introduction}

Wireless sensor networks (WSNs) are becoming a popular research area in recent years. They are considered as one of the most promising technologies due to their unique characteristics, low cost, easy deployment and flexibility. WSNs are employed in many applications such as medical monitoring, military, security, environment control and industry [1]. They are composed of numerous

I. Ez-zazi · M. Arioua · A. El Oualkadi

Laboratory of Information and Communication Technologies, National School of Applied Sciences, Abdelmalek Essaadi University, Tangier, Morocco

E-mail: imad.ezzazi@gmail.com,m.arioua@ieee.org, ahmed.eloualkadi@ieee.org

P. Lorenz

Network and Telecommunication Department, GRTC Laboratory, IUT-University of Haute

Alsace, Colmar, France

E-mail: lorenz@ieee.org 
smaller, cheaper and intelligent sensor nodes which are capable of sensing, processing and communicating the gathered information to the base station [2]. These sensor nodes are typically battery powered that can not be renewable for a long time [3]. Therefore, reducing energy consumption in order to prolong network lifetime is the most crucial design consideration in WSNs [4].

Communication reliability is another prime requirement in WSNs $[5,6]$. The increase of transmission energy results in successful and reliable transmission. However, the rise of transmission energy profoundly affects the sensor nodes energy and the network lifetime. It is worth noting that designing optimal WSN design implies providing reliable communication with the minimum required energy consumption. Error control coding (ECC) is an efficient method used to improve link reliability and lower the required transmission energy $[7,8]$. In low power application such as wireless sensor network, applying low power ECC techniques is primordial requirement [9-11]. Advanced coding schemes with complex decoding algorithms provide higher coding gain but require higher energy consumption. Thus, a trade-off between coding gain and decoder complexity should be considered in order to optimize the error correction schemes in WSN [12].

The reliability level provided by the link layer depends on the requirements of channel conditions for target applications [13]. Furthermore, channel conditions and inter-node distances are different as the WSN deployment environment varies. In practice, operating over short distances in multi-hop wireless sensor network enables significant energy consumption in decoding than transmission energy [14]. Moreover, the effect of path loss, multipath and fading phenomenon is unimportant by the reason of shortness of internode distances. When inter-node distances are long, the radio energy usage is significantly larger than decoding energy consumption and the effects of the path loss, multipath and fading phenomenon are dominant which affect the reliability of transmission as well as the energy consumption. Therefore, the substantial compromise between the decoding process and channel condition constraints leads to the proposition of an optimized hybrid scheme as using the same error control scheme in the entire network is not always an adequate choice. In this paper, we propose an implementation of a hybrid adaptive coding and decoding scheme in multi-hop WSN with the advanced LDPC codes at long distance transmission and soft RS codes in short distance transmission for effective trade-off between performance and energy consumption. Different adaptive control schemes have been proposed for wireless sensor networks [15, 16]. Compared with existing optimized schemes, the proposed method is effectively suitable for short and large multi-hop networks when both free space and multipath propagation are presented. Our goal in this work is to reduce transmit and decoding energy usage as well as improve transmission reliability in multi-hop WSNs.

The rest of the paper is organized as follows. The error correction coding approach is discussed in section 2 . In section 3 , the radio propagation model and energy model for wireless sensor network are discussed. In section 4 , the 
overall methodology and the proposed scheme are described. Simulations and results are presented in section 5 and finally, section 6 concludes the paper.

\section{Error correction coding in WSN}

Critical wireless sensor applications such as environment monitoring require the delivery of high priority events in a reliable and faster manner without any loss on the path from sensor nodes to the base station. However, the unstable nature of channels is the critical and unpredictable factor which disturbs the signal and causes data corruption [17]. All these constraints emphasize the need for reliability and robustness of the data transport and minimization of errors probability in spite of flawed channels [18]. Therefore, error correction coding is a prime solution for effective communications in WSN.

In error correction coding approach, errors are both detected and corrected at the receiver end. To enable the receiver to correct data, redundant information is combined with the original information at the transmitter. The capability of correcting errors induced by flawed channels in the received sequence is measured by providing better bit error rate (BER) performance for the same signal-to-noise ratio (SNR) compared to un-coded scheme or by providing the same BER at lower SNR than un-coded scheme [7]. The coding gain is the difference in required SNR to achieve a certain BER for a given coding scheme compared to un-coded scheme. Efficient coding schemes characterized by higher coding gain and less transmission energy.

Several error correction codes have been investigated for WSNs, including BCH codes, Reed Solomon (RS) codes, convolutional codes, Turbo codes and Low Density Parity Check (LDPC) codes [19-22]. RS codes have been proven suitable and energy efficient for wireless sensor network applications with simple and reasonable coding and decoding complexity [23]. Results presented in [24] show that using RS codes with short codeword length and BPSK modulation scheme in WSN saves about $50 \%$ of energy. LDPC codes have been proved to own better performance and several advantages over other block and convolutional codes [25]. They are the most performant coding schemes and competitors of Turbo codes in terms of performance and complexity. Experimental results in $[26,27]$ exhibit that LDPC codes are good candidates for WSN applications. However, LDPC codes require complex decoders with large hardware and energy consumption [20]. For this reason, authors of [26] proposed the use of short-length LDPC codes in WSN in order to mitigate the LDPC decoder effect and provide acceptable BER. An adaptive iterative decoding (AID) approach exploiting a threshold on the number of iterations of decoding algorithm for a certain BER is proposed in [28]. This approach with iterative LDPC decoding lowered the network energy consumption by $20-25 \%$. The extra power consumption of LDPC decoders limits the use of these codes in multi-hop WSN which lead authors of $[29,30]$ to suggest the use of efficient single-hop sensor network instead of multi-hop approach with powerful coding schemes. This approach considers the decoding only at the base station in or- 
der to save energy and prolong the network lifetime. Moreover, several works have been interested in the optimization and use of strong coding techniques in multi-hop network design. For instance, a low power consumption decodeand-forward multi-hop approach for WSN is proposed in [12], where a Viterbi decoding of convolutional codes is implemented in intermediate nodes while the iterative decoding algorithm of Turbo codes is applied at the base station.

In this paper, we investigate the use of advanced coding schemes in multihop WSN where each node encodes and decodes data at the transmission and reception respectively. Both the strong LDPC codes and soft RS codes are adaptively considered based on the channel conditions and inter-node distance.

\subsection{LDPC coding scheme}

LDPC codes are linear error correcting block codes described by a sparse $(M \times N)$ parity-check matrix $H$ [31]. The matrix $H$ can be represented as a bipartite graph known as Tanner Graph [32], with $M$ check nodes and $N$ variables nodes. Ones in the parity-check matrix $H$ are presented by edges in Tanner graph and most of decoding algorithms require the exchange of information along the edges of the graph. Belief Propagation (BP) algorithm is the most algorithm used to decode LDPC codes due to its performance [33]. The BP algorithm emphasizes that variable nodes update the received information from the channel according to the parity check equations evaluated at the check nodes. This process is repeated several times until either the maximum number of iterations is reached. The decoding complexity associated with $\mathrm{BP}$ algorithm is evaluated based on the number of real multiplications and additions processes in each iteration $[34,35]$.

$$
E_{L D P C}=\prod_{k=1}^{n}(3 N . i+6 t . N . j-10 N) E_{m u l t}+(3 N . j+N) E_{a d d}
$$

Where $t$ is the correction capacity, $N$ is the code word length, $i$ and $j$ are weight of row and column of parity check matrix for LDPC code, $n$ is the number of optimal iterations, $E_{a d d}$ and $E_{\text {mult }}$ denote the energy consumption per $m$-bit in the addition and multiplication, respectively, of field elements in Galoi Field $\left(G F\left(2^{m}\right)\right)$. These energy values are computed in [35] for $0.18 \mu \mathrm{m}$, $2.5 \mathrm{~V}$ CMOS based implementation.

\subsection{RS coding scheme}

RS codes are efficient error correction schemes specified by their relevant performance for reasonable decoding complexity. RS code is composed of $N$ symbols with a number of message symbols $k$ and redundant symbols $(N-\mathrm{k})$. It is characterized by a minimum distance of $d=N-\mathrm{k}+1$ and an error correction capacity of $t=(N-\mathrm{k}) / 2$. A relevant decoding technique for binary RS codes is based on the Berlekamp-Massey (BM) and Chien's search (CS) which present 
a linear dependence on block length $N$. The computation energy of RS codes based on BM and CS algorithms is expressed in [35] as:

$$
E_{R S}=\left(2 t . N+2 t^{2}\right)\left(E_{\text {mult }}+E_{\text {add }}\right)
$$

The complexity of the decoding increases with increasing the codeword length $N$ which require important amount of resources [26]. Moreover, the amount of sensed and exchanged data by sensor nodes in WSN applications is typically limited. In this context, we considered short block length LDPC and RS codes for error correction in WSN.

\section{Radio propagation \& Energy model}

\subsection{Radio propagation model}

Many radio propagation models known for wireless communications predict the signal-strength loss with distance-path loss. The free space and multipath propagation models are widely used for wireless sensor networks [36]. In the free space model, communications between the transmitter and the receiver are clear line-of-sight in which reflecting surfaces and effect of the earth surface are solely absent. The multipath propagation is as result of different signal paths between the transmitter and the receiver. These paths are the major detriment to signal propagation by giving rise to interference, distortion of the signal, loss of data and multipath fading.

For both free space and multipath fading, the received power decreases as the distance between the transmitter and receiver increases [37]. For this work, both the free space model and the multipath fading model were used, depending on the distance between the transmitter and receiver. If the inter-node distance (distance between transmitter and receiver) is less than a crossover distance $\left(d_{\text {crossover }}\right)$, the Friss free space model is used ( $d^{2}$ attenuation). Otherwise $\left(d>d_{\text {crossover }}\right)$, the two-ray ground propagation model is adopted $\left(d^{4}\right.$ attenuation). The crossover distance for Friss and two-ray-ground attenuation models is defined in [38] as:

$$
d_{\text {crossover }}=\frac{4 \pi \sqrt{L} h_{r} h_{t}}{\lambda}
$$

Where $L \geq 1$ is the system loss factor not related to propagation, $h_{r}$ and $h_{t}$ are the heights of the receiving and transmitting antennas above the ground respectively, and $\lambda$ is the transmitted wavelength corresponding to the transmitting frequency.

The transmit power is attenuated according to the Friss free space equation for distance bellow $d_{\text {crossover }}$ as follow:

$$
P_{r}(d)=\frac{P_{t} G_{t} G_{r} \lambda^{2}}{(4 \pi d)^{2} L}
$$


Where $P_{r}(d)$ is the receive power for a transmitter-receiver separation of inter-node distance d, $P_{t}$ is the transmit power, $G_{t}$ and $G_{r}$ are the gains of the transmitting and receiving antennas respectively and $d$ is the distance between the transmitter and receiver.

This attenuation is only considered when the transmitter and the receiver have direct line of sight communication, which occur when the inter-node distance is short $\left(d<d_{\text {crossover }}\right)$. For all distances greater than $d_{\text {crossover }}$, the transmit power is attenuated according to the two-ray ground propagation equation as follows:

$$
P_{r}(d)=\frac{P_{t} G_{t} G_{r} h_{t}^{2} h_{r}^{2}}{d^{4} L}
$$

In this work, we adopted the experiments described in [38] by using an omnidirectional antenna with the following parameters: $G_{t}=G_{r}=1, h_{t}=$ $h_{r}=1 m$ and no system loss $(\mathrm{L}=1)$. For low power WSN, we adopt the IEEE 802.15.4 standard with an operating frequency of $2.4 \mathrm{GHz}$ and $\lambda$ equals to $0.124 m$ [39]. Substituting these parameter values into Equation 3, the obtained crossover distance is the following:

$$
d_{\text {crossover }}=101 \mathrm{~m}
$$

\subsection{Radio energy model}

The first order radio energy model is adopted for our studies [38,40,41]. This model considers the energy consumed at the transmitter and the receiver to send and receive an $L$-bits message over a distance $d$ (Figure 1 ). In sensor nodes, the transmitter dissipates energy to run the radio electronics $E_{T x-e l e c}$ and the amplifier energy $E_{T x-a m p}$ and the receiver dissipates energy to run the radio electronics. Thus, the energy expended to transmit an $L$-bits message over a distance $d$ is described as:

$$
E_{T x}(L, d)=E_{T x-e l e c}(L)+E_{T x-a m p}(L, d)
$$

Considering free space and multipath propagation, the equation 6 is formulated as:

$$
E_{T x}(L, d)= \begin{cases}E_{\text {elec }} \cdot L+\epsilon_{\text {friss-amp. }} \cdot L \cdot d^{2} & \text { if } d<d_{\text {crossover }} \\ E_{\text {elec }} \cdot L+\epsilon_{\text {two-ray-amp }} \cdot L \cdot d^{4} & \text { if } d \geq d_{\text {crossover }}\end{cases}
$$

When receiving data, the energy expended is expressed as:

$$
E_{R x}(L)=E_{\text {elec }} \cdot L
$$

Where $E_{\text {elec }}$ corresponds to the energy per bit required in transmit and receive electronics to process the information.

The parameters $\epsilon_{\text {friss-amp }}$ and $\epsilon_{t w o-r a y-a m p}$ depend on the required received sensitivity and the receiver noise figure (RNF), as the transmit power 


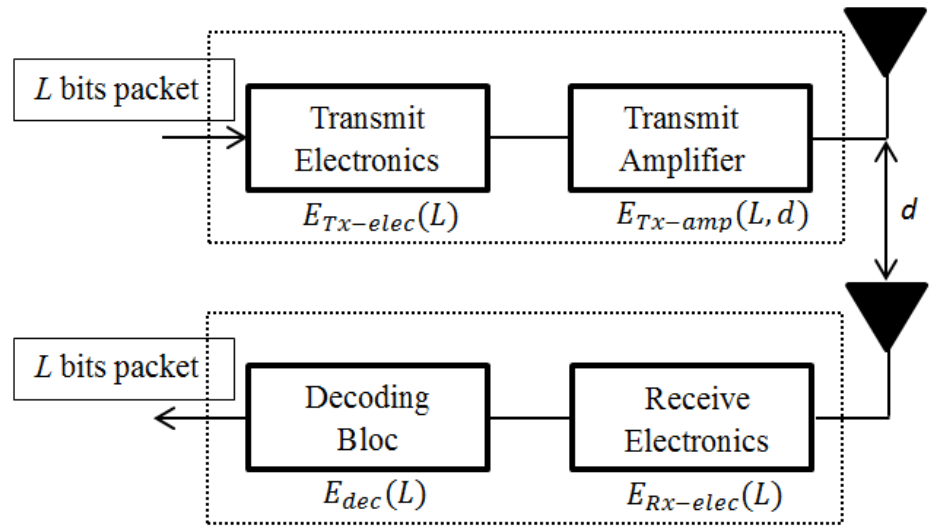

Fig. 1 First order radio energy model with decoder bloc

has to be adapted so that the power at the receiver is above a certain threshold $P_{r-t h r e s h}$. The minimum transmit power is determined based on this receive power threshold. If the radio bit rate is $R$, the transmit power is equal to the transmit energy per bit $E_{T x-a m p}(1, d)$ multiplied by the bit rate $R$.

$$
P_{t}=E_{T x-a m p}(1, d) \cdot R
$$

Substituting in the value of $E_{T x-a m p}(1, d)$ gives:

$$
P_{t}= \begin{cases}\epsilon_{\text {friss-amp. }} \cdot R \cdot d^{2} & \text { if } d<d_{\text {crossover }} \\ \epsilon_{\text {Two-ray-amp }} \cdot R \cdot d^{4} & \text { if } d \geq d_{\text {crossover }}\end{cases}
$$

Using Equations 4 and 5, the received power is expressed as:

$$
P_{r}= \begin{cases}\frac{\epsilon_{\text {friss-amp }} \cdot R \cdot G_{t} \cdot G_{r} \cdot \lambda^{2}}{(4 \pi)^{2}} & \text { if } d<d_{\text {crossover }} \\ \epsilon_{\text {Two-ray-amp }} \cdot R \cdot G_{t} \cdot G_{r} \cdot h_{t}^{2} \cdot h_{r}^{2} & \text { if } d \geq d_{\text {crossover }}\end{cases}
$$

The parameters $\epsilon_{\text {Friss-amp }}$ and $\epsilon_{\text {Two-ray-amp }}$ are determined by setting equation 11 equals to $P_{r-t h r e s h}$ :

$$
\begin{aligned}
& \epsilon_{\text {Friss-amp }}=\frac{P_{r-t h r e s h} \cdot(4 \pi)^{2}}{R \cdot G_{t} G_{r} \lambda^{2}} \\
& \epsilon_{\text {Two-ray-amp }}=\frac{P_{r-t h r e s h}}{R \cdot G_{t} G_{r} h_{t}^{2} h_{r}^{2}}
\end{aligned}
$$

The required transmit power $P_{t}$ as a function of the receiver threshold and the distance between the transmitter and receiver is determined by substituting equations 12 and 13 in equation 10 :

$$
P_{t}= \begin{cases}\frac{(4 \pi)^{2}}{G_{t} G_{r} \lambda^{2}} \cdot P_{r-\text { thresh }} \cdot d^{2} & \text { if } d<d_{\text {crossover }} \\ \frac{1}{G_{t} G_{r} h_{t}^{2} h_{r}^{2}} \cdot P_{r-t h r e s h} \cdot d^{4} & \text { if } d \geq d_{\text {crossover }}\end{cases}
$$


The receiver threshold $P_{r-t h r e s h}$ is determined by estimating the noise at the receiver. Considering the thermal noise floor equals to $99 \mathrm{dBm}$, the receiver noise figure of $17 \mathrm{~dB}$ and the required signal-to-noise ratio (SNR) of at least $30 \mathrm{~dB}$ to receive the signal with no errors [38], the minimum receive power $P_{r-t h r e s h}$ for successful reception is determined as:

$$
P_{r-\text { thresh }} \geq 30+(-82)=-52 \mathrm{dBm}
$$

Accordingly, the received power must be at least $-52 \mathrm{dBm}$ or $6.3 \mathrm{nW}$ for successful reception of the packet [38]. Considering the experiment parameters used in this work $\left(G_{t}=G_{r}=1, h_{t}=h_{r}=1 m, \lambda=0.124\right)$, and as the IEEE 802.15.4 standard target a throughput $(R=250 \mathrm{kbps})$, the equations 12 and 13 are expressed as:

$$
\left\{\begin{array}{l}
\epsilon_{\text {Friss-amp }}=2.59 * 10^{-10} \mathrm{~J} / \mathrm{bit} / \mathrm{m}^{2} \\
\epsilon_{\text {Two-ray-amp }}=2.52 * 10^{-14} \mathrm{~J} / \mathrm{bit} / \mathrm{m}^{4}
\end{array}\right.
$$

\subsection{Energy model for multi-hop WSN}

The energy model for multi-hop wireless sensor networks using error correction codes takes into account decoding energy for all nodes except source node. Energy consumed in encoding is considered for all nodes except the last node. However, encoding in block codes is typically a simple process with insignificant computational energy [9]. Thus, the encoding energy is not considered in the energy model.

The total energy required to transmit, receive and decode information is given as:

$$
E_{T o t}=\sum_{1}^{m} E_{T x}+\sum_{1}^{m-1} E_{R x}+\sum_{1}^{m-1} E_{R x-D e c}
$$

Where $E_{T o t}$ is the total energy consumed in the multi-hop network, $E_{T x}$ is the energy consumed in transmission by all nodes, $E_{R x}$ is the energy consumed in receiving information by all nodes except source node, $E_{R x-D e c}$ is the energy consumed in decoding process by all nodes except source node, $m$ is the number of hopes and $L$ is the packet size in bits.

In Minimum Transmission Energy (MTE) algorithm, nodes route data destined for the base station through intermediate nodes. These nodes act as a routers of other nodes data in addition to sensing the environment. Each data message must go through $m$ transmits a distance $r$ and $m-1$ receives. Thus, the total radio energy is expressed as:

$$
E_{M T E}=m \cdot E_{T x}(L, d=r)+(m-1) E_{R x}(L)
$$

For free space propagation: 


$$
\begin{aligned}
E_{M T E} & =m\left(E_{\text {elec }} \cdot L+\epsilon_{\text {friss-amp }} \cdot L \cdot r^{2}\right)+(m-1) E_{\text {elec }} \cdot L \\
& =L\left((2 m-1) E_{\text {elec }}+\epsilon_{\text {friss-amp }} \cdot m \cdot r^{2}\right)
\end{aligned}
$$

For multipath propagation:

$$
\begin{aligned}
E_{M T E} & =m\left(E_{\text {elec }} \cdot L+\epsilon_{t w o-r a y-a m p} \cdot L \cdot r^{4}\right)+(m-1) E_{\text {elec }} \cdot L \\
& =L\left((2 m-1) E_{\text {elec }}+\epsilon_{t w o-r a y-a m p} \cdot m \cdot r^{4}\right)
\end{aligned}
$$

\section{Proposed scheme}

The main goal of the proposed scheme is to prolong energy lifetime as well as improve the reliability of communications in multi-hop wireless sensor networks. This is achieved by protecting data transmission from noise, avoiding extra transmissions, interferences, multipath fading and packet loss. Selection of the adequate error control coding usually depends on the distance and the transmission environment [7]. Noisy environment with fading, shadowing and multipath typically requires more efficient control coding with strong decoding scheme to retrieve all data correctly. LDPC codes are the most efficient codes in the area used for reliable communication with BER performance closed to the Shannon limit $[26,42]$. However, these codes employ a complex iterative decoding in the receiver which raises the energy consumption. The suggested scheme is a hybrid adaptive algorithm aims to optimize the decoding usage in multi-hop network. In fact, it benefits from the efficiency and potency of LDPC code in multipath environment. While, in reliable free space environment it employs an efficient coding scheme as RS code with simple decoding algorithm. Through this combination, we aspire to minimize the energy computation consumed by decoders and ensure substantial reliability and flexibility.

We employed MTE routing algorithm to minimize the inter-nodes propagation distance in order to frequently provide free space routes than multipath propagation. Each node in the considered network sends their information with MTE routing by using the nearest neighbor in the optimum path toward the base station. A simple network of multi-hop communication is shown in Figure 2 , in which $n$ nodes are separated a distance $r$ apart from each other.

Inter-node distances depend on different applications and vary from few to hundreds of meters. For instance, in a WSN deployed for glacial environment monitoring [43], nodes were kept 20-25 $m$ apart, whereas for volcano monitoring [44], nodes were deployed 200-400 $m$ apart. Nodes typically start with an important energy and keep near each other few meters apart and most of communications are successfully performed. After a certain time, nodes begin to die due to loss of energy and inter-node distances become longer which cause detriment and loss of communications.

The realized scheme takes into account inter-node distances and channel conditions to decide adequate error correction code. RS and LDPC codes are 


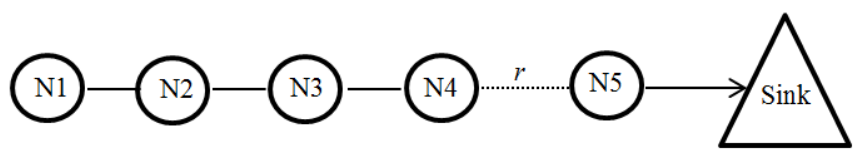

Fig. 2 Muti-hop network of n nodes

jointly applied to provide error protection on the transmitted data. RS code is operational when inter-node communication occurred over a distance $d$ lower than the crossover distance. On the other hand, LDPC code is integrated and used when inter-node distance values exceed $d_{\text {crossover }}$. Therefore, RS coding/decoding scheme is exclusively adopted in direct propagation and LDPC coding/decoding scheme when the multipath propagation model is selected. Figure 3 presents the mechanism of the implemented coding and decoding scheme.

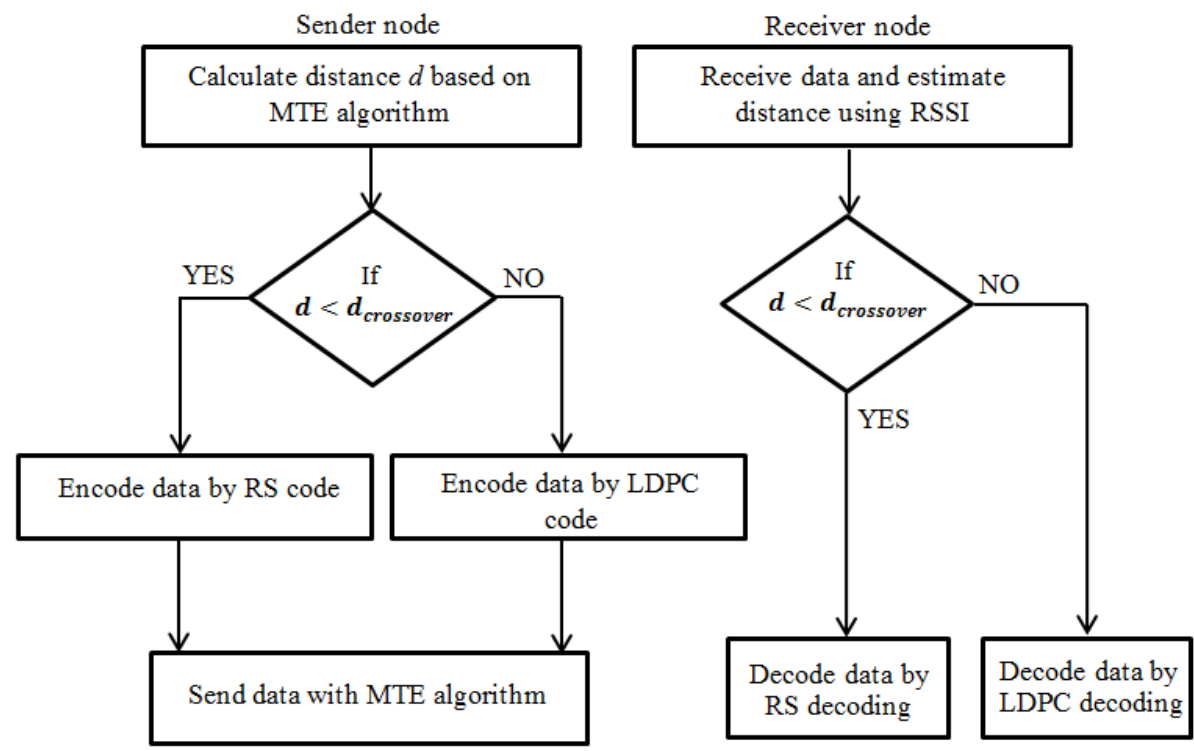

Fig. 3 Proposed error correction scheme for sender and receiver node

Using equations (1), (2), (19) and (20) of radio and decoder energy models, we obtain the total energy of the system for both RS and LDPC schemes as follow: 


$$
E_{T o t}=E_{T o t-R S}+E_{T o t-L D P C}
$$

Where:

$$
\begin{array}{r}
E_{\text {Tot-RS }}=L\left((2 m-1) E_{\text {elec }}+\epsilon_{\text {friss-amp }} \cdot m \cdot r^{2}\right) \\
+\left(2 t . N+2 t^{2}\right)\left(E_{\text {mult }}+E_{\text {add }}\right)
\end{array}
$$

And

$$
\begin{aligned}
& E_{T o t-L D P C}=L\left((2 m-1) E_{\text {elec }}+\epsilon_{t w o-r a y-a m p} \cdot m . r^{4}\right) \\
& +\prod_{k=1}^{n}(3 N . i+6 t . N . j-10 N) E_{\text {mult }}+(3 N . j+N) E_{\text {add }}
\end{aligned}
$$

Nodes adaptively decide the adequate error correction code to use according to distance and channel conditions. At the sender, nodes calculate and choose the optimum distance to send their data using MTE algorithm. At the receiver, nodes have to estimate the distance from the sender in order to decide which decoding algorithm has to be used. Received signal strength indicator (RSSI) is a measurement of the power presented in the received radio signal $[45,46]$. When data packet is received, the value of RSSI feedback is used to estimate the distance from the transmitter as well as the path-loss phenomenon. Based on RSSI indicator, nodes decide which decoding scheme will be employed. The choice of the adequate coding and decoding method takes into account two crucial parameters: reliability of transmission and energy efficiency.

\section{Simulation and results}

MATLAB simulator tool is used to analyze the impact of the hybrid adaptive coding and decoding scheme on the energy of the entire network. For all simulations referred in our work, we followed the energy consumption models and parameters of reference $[38,40]$ and we used the values for the energy consumption related parameters as shown in Table 1. A packet size of $L=2000$ bits is used, 50 sensors are randomly deployed in a network dimensions of $50 \mathrm{~m}$ $\mathrm{x} 50 \mathrm{~m}$ and $200 \mathrm{~m} \times 200 \mathrm{~m}$. The base station is placed at $(x=25 \mathrm{~m}, y=-100 \mathrm{~m})$ and $(x=100 \mathrm{~m}, y=-100 \mathrm{~m})$, respectively. All nodes begin with a starting energy level of $1 \mathrm{~J}$. This value is commonly used in the literature since it provides small enough energy to quickly see the effect of the varying algorithms. In our simulations, MTE algorithm does not employ data aggregation strategy due to the fact that at each round every node is assumed to transmit its message in a TDMA scheme where there is only one message passing from source node to base station through the network at a time.

Concept of transmit power is used with respect of distance, less distance requires less transmit power while large distance requires greater transmit 
Table 1 Simulation parameters

\begin{tabular}{c|c}
\hline Parameter & Value \\
\hline \hline Tx Amplifier, free space propagation, $\epsilon_{f s}$ & $2.59^{*} 10^{-10} \mathrm{~J} / \mathrm{bit} / \mathrm{m}^{2}$ \\
Tx Amplifier, multipath propagation, $\epsilon_{m p}$ & $2.52^{*} 10^{-14} \mathrm{~J} / \mathrm{bit} / \mathrm{m}^{4}$ \\
Tx and Rx energy electronics $E_{e l e c}$ & $50 \mathrm{~nJ} / \mathrm{bit}$ \\
Initial node energy (Joules) & $1 \mathrm{~J}$ \\
Number of nodes & 50 \\
Frequency & $2.45 \mathrm{GHz}$ \\
Bit rate & $250 \mathrm{kbps}$ \\
Antennas gain & $1 \mathrm{~dB}$ \\
high of antennas & $1 \mathrm{~m}$ \\
$d_{\text {crossover }}$ & $101 \mathrm{~m}$ \\
Packet size & $2000 \mathrm{bits}$ \\
Addition energy $E_{\text {add }}$ & $3.3^{*} 10^{-5} \mathrm{~m}(\mathrm{~mW} / \mathrm{MHz})$ \\
Multiplication energy $E_{m u l t}$ & $3.7^{*} 10^{-5} m^{3}(\mathrm{~mW} / \mathrm{MHz})$ \\
\hline
\end{tabular}

power. The total system energy versus transmission round in $50 \mathrm{~m}^{2}$ and $200 \mathrm{~m}^{2}$ network is shown in Figures 4 and 5, respectively. Obtained simulations display the effect of distance and decoder complexity on total energy system and nodes lifetime. Increasing the inter-node distances, the total energy of the network is hastily consumed and most of nodes die quickly. In $50 \mathrm{~m}^{2}$ sensor network, the simulation shows that the energy of the network with the un-coded scheme is completely consumed in 1514 rounds. The energy of the network implementing LDPC coding scheme is fully depleted in 1136 rounds which correspond to lifetime decrease of $25 \%$ when compared to the un-coded scheme. The energy of the network with RS coding scheme is completely dissipated in 1357 rounds which correspond to lifetime decrease of $10 \%$. As expected, system with RS decoding is more energetically efficient than LDPC decoding scheme. Using LDPC decoding in the entire network intensely consumes and drains nodes energy. By using the proposed coding scheme, the energy of sensor network is wholly consumed in 1315 rounds which correspond to lifetime increase of $15 \%$ compared to LDPC coded scheme. In $200 \mathrm{~m}^{2}$ sensor network with uncoded scheme, the total energy is completely consumed in 272 rounds. For LDPC coded scheme the energy is totally depleted in 213 rounds which correspond to lifetime decrease of $27 \%$ when compared to uncoded scheme. For the RS coded scheme, the energy is dissipated in 244 rounds which corresponds to lifetime decrease of $10 \%$ when compared to uncoded scheme. Apparently, by adopting the proposed coding scheme, the energy of sensor network is wholly consumed in 237 rounds which correspond to lifetime increase of $11 \%$ compared to LDPC coded scheme.

Figures 6 and 7 show the number of nodes alive in the case of unocoded scheme, RS coded scheme, LDPC coded scheme and proposed hybrid adaptive scheme in $50 \mathrm{~m}^{2}$ and $200 \mathrm{~m}^{2}$ network dimensions. Table 2 summarizes the round when $10 \%, 50 \%$ and $90 \%$ of nodes are dead in the networks. In 50 


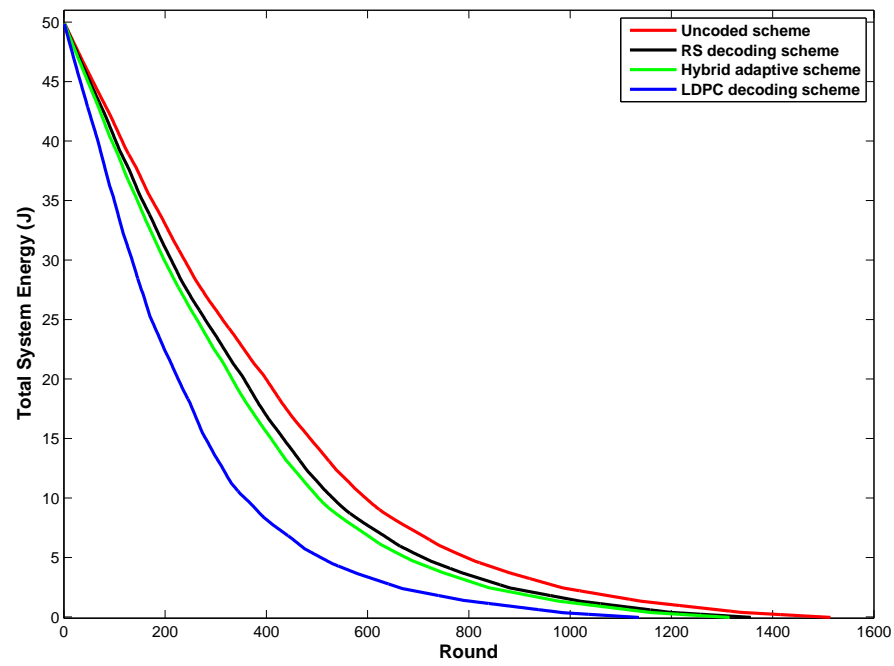

Fig. 4 Total system energy over $50 \mathrm{~m}^{2}$ sensor network

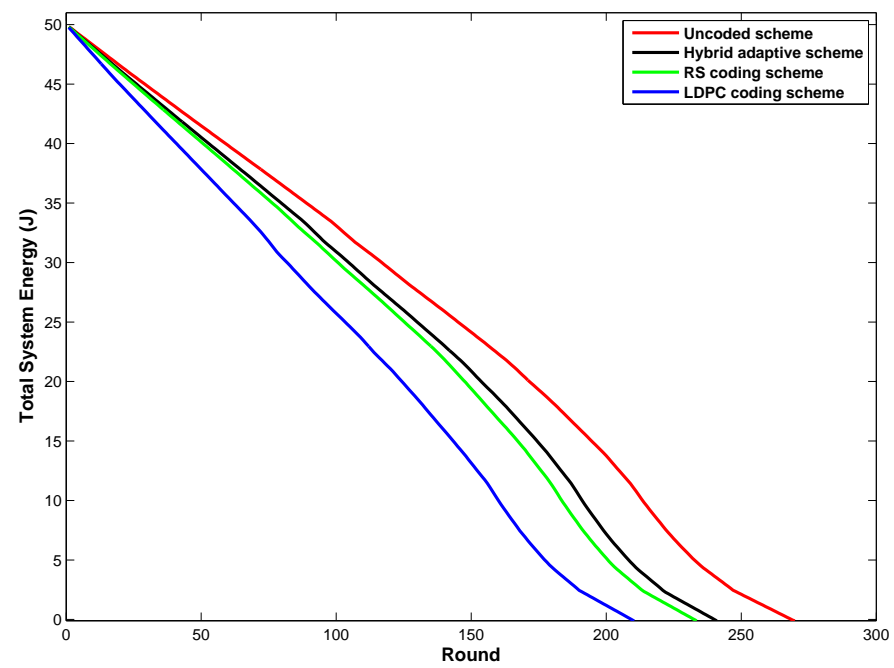

Fig. 5 Total system energy over $200 \mathrm{~m}^{2}$ sensor network

$m^{2}$ sensor network, when the WSN system transmits LDPC coded data, the percentages of $10 \%, 50 \%$ and $90 \%$ of dead nodes occur at 98 rounds, 282 rounds and 580 rounds, respectively. Using the proposed hybrid adaptive scheme, the percentages of 10\%, 50\% and 90\% of dead nodes occur at 108 rounds, 395 rounds and 748 rounds, respectively. This corresponds to lifetime increase of $10 \%, 40 \%$ and $28 \%$, respectively when compared to LDPC coded scheme. the proposed coding scheme outperformed LDPC coding scheme by optimizing the nodes lifetime of $26 \%$ approximately. In $200 \mathrm{~m}^{2}$ sensor network with LDPC 
coding scheme, the percentages of $10 \%, 50 \%$ and $90 \%$ of dead nodes occur at 68 rounds, 136 rounds and 181 rounds, respectively. In this case, the proposed coding scheme improves the nodes lifetime of $16 \%, 14 \%, 13 \%$, respectively when compared to LDPC coded scheme. Accordingly, the proposed hybrid adaptive coding scheme outclasses the LDPC coding scheme in both $50 \mathrm{~m}^{2}$ and $200 \mathrm{~m}^{2}$.

Adopting the proposed hybrid adaptive scheme efficiently saves the energy batteries of sensor nodes and extends the network lifetime by saving the energy of nodes consumed in the decoding process. In addition, the proposed coding framework improves the communication reliability by adaptively using the RS scheme in free space propagation and the LDPC scheme in multipath propagation. The aggregated data transmitted from nodes are decoded using the BP decoding of LDPC code in the base station which result in effective decoding and retrieving of the sensed data.

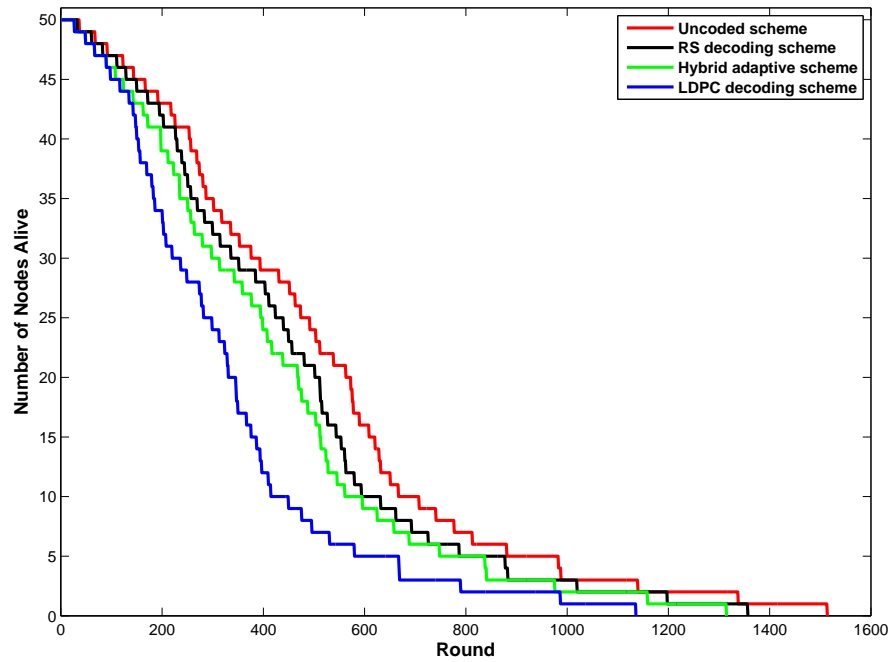

Fig. 6 Number of nodes alive over $50 \mathrm{~m}^{2}$ sensor network

Table 2 Overall algorithm die-off statistics of LDPC and proposed schemes in $50 \mathrm{~m}^{2}$ and $200 \mathrm{~m}^{2}$ networks

\begin{tabular}{l|l|l|l|l|l|l}
\hline Coding scheme & \multicolumn{2}{|c|}{ LDPC scheme } & \multicolumn{2}{l|}{ Proposed scheme } & \multicolumn{2}{c}{$\%$ increase } \\
\hline Network dimension & $50 \mathrm{~m}^{2}$ & $200 \mathrm{~m}^{2}$ & $50 \mathrm{~m}^{2}$ & $200 \mathrm{~m}^{2}$ & $50 \mathrm{~m}^{2}$ & $200 \mathrm{~m}^{2}$ \\
\hline \hline $10 \%$ Nodes Dead & 98 & 68 & 108 & 79 & 10 & 16 \\
$50 \%$ Nodes Dead & 282 & 136 & 395 & 156 & 40 & 14 \\
$90 \%$ Nodes Dead & 580 & 181 & 748 & 205 & 28 & 13 \\
\hline
\end{tabular}




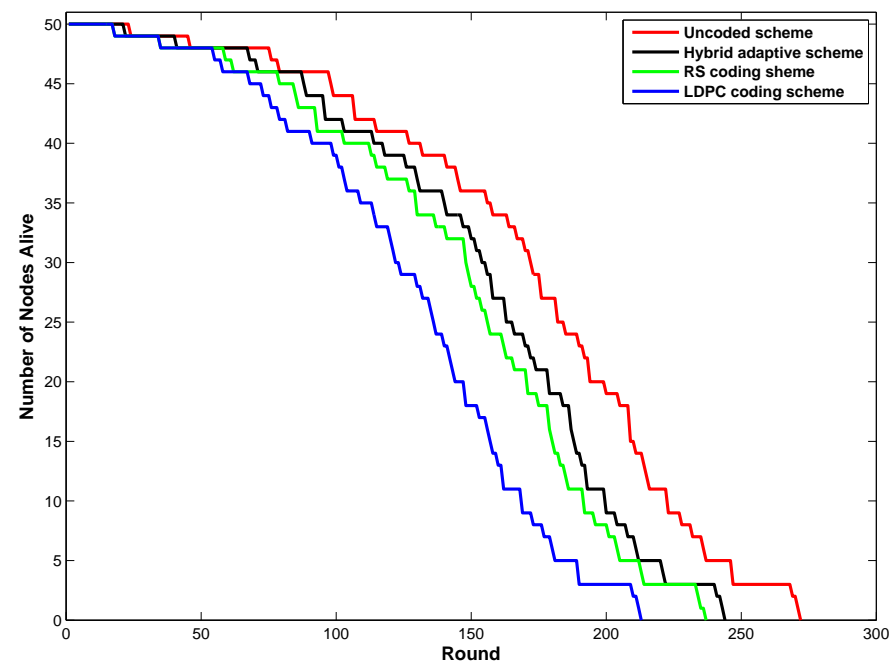

Fig. 7 Number of nodes alive over $200 \mathrm{~m}^{2}$ sensor network

\section{Conclusion}

In this paper, we have proposed a hybrid adaptive coding and decoding scheme based on inter-node distances and channel conditions to improve network lifetime and multi-hop transmission reliability. Extensive simulations have been carried out to demonstrate the advantages of the proposed method based on $\mathrm{RS}$ and LDPC codes. The results have shown that the new adaptive approach improves both energy consumption and communication reliability in the multihop wireless sensor networks.

This hybrid adaptive scheme can be adopted for different types of networks. The evolution and enhancement of the presented scheme should be done in the future to optimize energy consumption and transmission reliability in a clustered wireless sensor networks.

\section{References}

1. H. M. Ahmad Fahmy (2016) . WSNs Applications. Wireless Sensor Networks, Springer, 69-213

2. Ammari H. M., Das S. K. (2012). Centralized and clustered k-coverage protocols for wireless sensor networks. IEEE Transactions on Computers, 61, 118-133.

3. S. Yadav, R. S. Yadav (2015). A review on energy efficient protocols in wireless sensor networks. Wireless Networks, 22(1), 335-350

4. H.W. Kim, A. Kachroo, (2016). Low power routing and channel allocation of wireless video sensor networks using wireless link utilization. Ad Hoc and Sensor Wireless Networks, 30, 83-112.

5. D.-W. Lee, J.-H. kim (2010). High reliable in-network data verification in wireless sensor networks. Wireless Personal Communications, 54, 501-519

6. M. A. Mahmood, W.K.G. Seah and I. Welch (2015). Reliability in wireless sensor networks: A survey and challenges ahead. Computer Networks (Elsevier), 79, 166-187 
7. S. L. Howard, C. Schlegel, K. Iniewski (2006). Error control coding in low-power wireless sensor networks: When is ECC energy-efficient?. EURASIP Journal on Wireless Communications and Networking, 2006, 1-14.

8. Tallal Elshabrawy (2015). Network throughput analysis of IEEE 802.15.4 enabled wireless sensor networks with FEC coding under external interference. International Journal of Electronics and Communications (AEU), Elsevier, 69(11), 1641-1649.

9. M. C. Vuran, I. F. Akyildiz (2009). Error control in wireless sensor networks: a cross layer analysis. IEEE/ACM Transactions on Networking, 17, 1186-1199.

10. Ali Alrajeh N., Marwat U., Shams B., Shah S.S.h. (2015). Error Correcting Codes in Wireless Sensor Networks: An Energy Perspective. Applied Mathematics and Information Sciences, 9(2), 809-818.

11. P. Salija and B. Yamuna (2015). Optimum energy efficient error control techniques in wireless systems: a survey. Journal of Communications Technology and Electronics, 60(11), 1257-1263.

12. N. Abughalieh, K. Steenhaut, A Nowe, A. Anpalagan (2014) . Turbo codes for multihop wireless sensor networks with decode-and-forward mechanism. EURASIP Journal on Wireless Communications and Networking, 2014(1), 1-13.

13. P. Padilla, J. Camacho, G. Macia-Fernandez, J. E. Diaz-Verdejo, P. Garcia-Teodoro, C. Gomez-Calero (2012). On the Influence of the Propagation Channel in the Performance of Energy-Efficient Geographic Routing Algorithms for Wireless Sensor Networks (WSN). Wireless Personal Communications, 70, 15-38.

14. S. Chouhan, R. Bose, M. Balakrishnan (2009). Framework for energy consumption based design space exploration for wireless sensor nodes. IEEE Transaction on Computer-Aided Design of Integrated Circuits and Systems, 28, 1017-1024.

15. Kleinschmidt J. H., Borelli W. C., Pellenz M. E. (2007). An analytical model for energy efficiency of error control schemes in sensor networks. International Conference on Communications, 3895-3900.

16. Joao H. Kleinschmidt, Walter C. Borelli, Marcelo E. Pellenz (2009). An energy efficiency model for adaptive and custom error control schemes in Bluetooth sensor networks. AEUInternational Journal of Electronics and Communications (Elsevier), 63, 188-199.

17. V. Nithya, B. Ramachandran, V. Bhaskar (2014).Energy Efficient Coded Communication for IEEE 802.15.4 Compliant Wireless Sensor Networks. Wireless Personal Communications, 77, 675-690.

18. Z. I. Kiss, Z. A. Polgar, M. P. Stef and V. Bota (2014). Improving transmission reliability in wireless sensor networks using network coding. Telecommunication Systems, 59(4), 509521

19. Pellenz M. E., Souza R. D., Fonseca M. S. P. (2010). Error control coding in wireless sensor networks. Journal of Telecommunication Systems, 44, 61-68.

20. A. D. G. Biroli, M. Martina, G. Masera (2012). An LDPC Decoder Architecture for Wireless Sensor Network Applications. Sensors Journal, 12, 1529-1543.

21. Liang Li, Maunder R.G., Al-Hashimi B.M., Hanzo, L. (2013) . A Low-Complexity Turbo Decoder Architecture for Energy-Efficient Wireless Sensor Networks. IEEE Transactions on Very Large Scale Integration (VLSI) Systems, 21, 14-22.

22. Soude H, Agueh M, Mehat J (2009). Towards an optimal Reed Solomon codes selection for sensor networks. In Proceedings of the 6th ACM Symposium on Performance Evaluation of Wireless Ad Hoc, Sensor, and Ubiquitous Networks. ACM Press, 165-166.

23. I.Ez-zazi, M.Arioua, A.El Oualkadi, Y.El Assari (2015). Performance analysis of efficient coding schemes for wireless sensor networks. Proceedings of IEEE international Workshop on RFID and Wireless Sensor Network RAWSN, 42-47.

24. S. Chouhan, R. Bose, M. Balakrishnan (2009). Integrated energy analysis of error correcting codes and modulation for energy efficient wireless sensor nodes. IEEE Transactions on Wireless Communications, 8(10), 5348-5355.

25. T. Richardson, R. Urbanke (2001). Efficient encoding of low-density parity-check codes. IEEE Transaction on Information Theory, 47, 638-656.

26. Duc Minh Pham, Syed Mahfuzul Aziz (2014), On efficient design of LDPC decoders for wireless sensor networks. Journal of networks, 9, 3207-3214.

27. S.-M. Choi, B.-H. Moon (2011). Implementation of Energy Efficient LDPC Code for Wireless Sensor Node. Communication and Networking, Springer, 248-257. 
28. N. Javaid, O. Rehman, N. Alrajeh, Z. A. Khan, B. Manzoor, S. Ahmed (2013). AID: An Energy Efficient Decoding Scheme for LDPC Codes in Wireless Body Area Sensor Networks. International Workshop on Communications and Sensor Networks, 449-554.

29. Schmidt D, Berning M, Wehn N (2009). Error correction in single-hop wireless sensor networks - a case study. In Design, Automation, and Test in Europe Conference and Exhibition, 1296-1301, Nice.

30. Zhong LC, Rabaey JM, Wolisz A (2005). Does proper coding make single hop wireless sensor networks reality: the power consumption perspective. IEEE Wireless Communications and Networking Conference. USA, 664-669.

31. Gallager, R.G. (1962). Low-density parity-check codes. IRE Transaction on Information Theory, 8, 21-28.

32. Tanner, R.M. (1981). A recursive approach to low complexity codes. IEEE Transaction on Information Theory, 27(9), 533-548.

33. MacKay, D.J.C. (2002). Good error-correcting codes based on very sparse matrices. IEEE Transaction on Information Theory, 45, 399-431.

34. Sang-Min Choi, Byung-Hyun Moon (2006). Performance Analysis on Wireless Sensor Network using LDPC Codes over Node-to-node Interference. proceedings of the International Technical Conference on Circuits Systems, Computers and Communications(ITCCSCC), 3, 461-464.

35. Y. Sankarasubramaniam, I. F. Akyuildiz, S. W. McLaughin (2003). Energy efficiency based packet size optimization in wireless sensor networks. Proceedings of IEEE International Workshop on Sensor Network Protocols and Applications SNPA'03, 1-8.

36. Joao C. Giacomin, Luiz H. A. Correia, Tales Heimfarth, Geraldo M. Pereira, Vinicius F. Silva, Joao L. P. De Santana (2010). Radio Channel Model of Wireless Sensor Networks Operating in $2.4 \mathrm{GHz}$ ISM Band. INFOCOMP Journal of Computer Science, 9.

37. T. Rappaport (1996). Wireless communications: Principles and practice. Prentice-Hall, Inc., New Jersey

38. W. B. Heinzelman (2000). Application-Specific Protocol Architectures for Wireless networks. Massachusetts Institute of technology.

39. Li, L., Maunder R.G., Al-Hashimi B.M., Hanzo L. (2010). An Energy-Efficient Error Correction Scheme for IEEE 802.15.4 Wireless Sensor Networks. IEEE Transactions on Circuits and Systems II , 57, 233-237.

40. W. B. Heinzelman, A. P. Chandrakasan, H. Balakrishnan (2002). An application specific protocol architecture for wireless microsensor networks. IEEE Transactions on Wireless Communications, 1, 660-670.

41. Qin Lu, Wusheng Luo, Jidong Wang and Bo Chen (2008). Low-complexity and energy efficient image compression scheme for wireless sensor networks. Computer Networks (Elsevier), 52(13), 2594-2603.

42. M. K. Roberts, R. Jayabalan (2014). An Improved Low Complex Hybrid Weighted BitFlipping Algorithm for LDPC Codes. Wireless Personal Communications, 82, 327-339.

43. K. Martinez, P. Padhy, A. Riddoch, H. Ong, J. K. Hart (2005). Glacial environment monitoring using sensor networks. Real-World Wireless Sensor Networks Workshop, 10-14.

44. G. Werner-Allen, K. Lorincz, M. Welsh, O. Marcillo, J. Johnson, M. Ruiz, and J. Lees (2006). Deploying a wireless sensor network on an active volcano. IEEE Internet Computing Journal, 10, 18-25.

45. K. Benkic, M. Malajner, P. Planinsic, Z. Cucej (2008). Using RSSI value for distance estimation in wireless sensor networks based on ZigBee. International IEEE Conference of Systems, Signals and Image Processing (IWSSIP), 303-306.

46. Miroslav botta, Milan simek (2013). Adaptive Distance Estimation Based on RSSI in 802.15.4 Network. Radioengineering journal, 22, 1162-1168. 

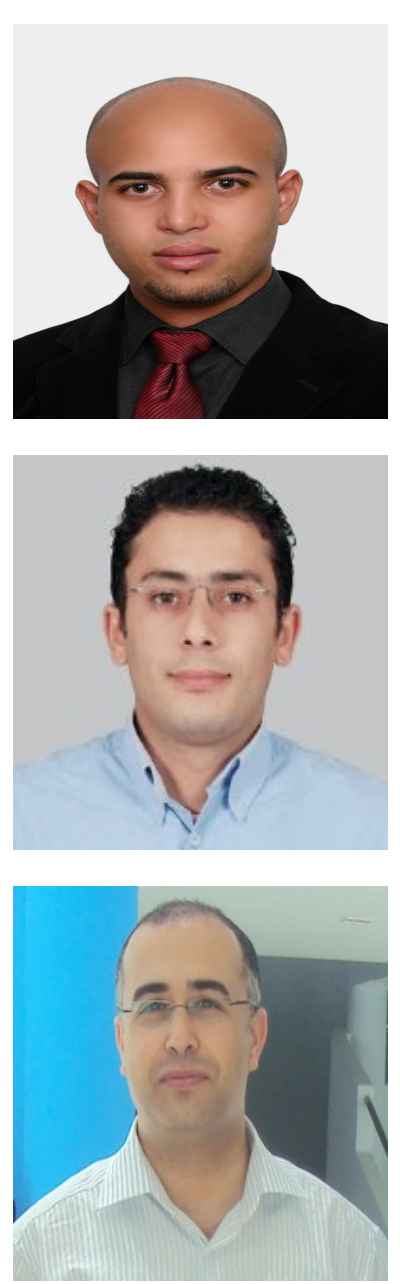

Ahmed EL OUALKADI received Ph.D degree from Poitiers University, France, in 2004. From 2000 to 2003, he was a research assistant at the National Higher Engineering School of Poitiers. In 2004, he was an assistant professor at University Institute of Technology, Angoulme. In 2005, he joined the Catholic University of Louvain, Belgium. Currently, he is an associate professor at national school of applied sciences of Tangier, Abdelmalek Essaadi University. His main research interest is RFIC design for wireless communication, wireless sensor networks, embedded system applications and information technology.

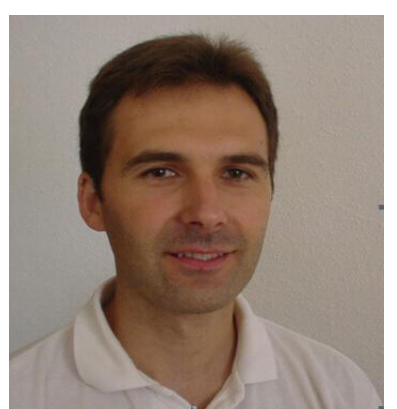

Pascal LORENZ received a $\mathrm{Ph} . \mathrm{D}$ degree from the University of Nancy, France. Between 1990 and 1995 he was a research engineer at WorldFIP Europe and at AlcatelAlsthom. He is a professor at the University of Haute-Alsace and responsible of the Network and Telecommunication Research Group. His research interests include wireless sensor networks, QoS, wireless networks and high-speed networks. $\mathrm{He}$ is the author/co-author of 3 books, 3 patents and 200 international publications in refereed journals and conferences. 